\title{
Fisher Distribution for Texture Modeling of Polarimetric SAR Data
}

\author{
Lionel Bombrun, Student Member, IEEE, and Jean-Marie Beaulieu
}

\begin{abstract}
The multi-look polarimetric SAR covariance matrix is generally modeled by a complex Wishart distribution. For textured areas, the product model is used and the texture component is modeled by a Gamma distribution. In many cases, the assumption of Gamma distributed texture is not appropriate. The Fisher distribution does not have this limitation and can represent a large set of texture distributions. As an example, we examine its advantage for an urban area. From a Fisher distributed texture component, we derive the distribution of the complex covariance matrix for multi-look polarimetric SAR data. The obtained distribution is expressed in term of the KummerU confluent hypergeometric function of the second kind. Those distributions are related to the Mellin transform and second kind statistics (Log-statistics). The new KummerU based distribution should provide in many cases a better representation of textured areas than the classic $\mathcal{K}$ distribution. Finally, we show that the new model can discriminate regions with different texture distribution in a segmentation experiment with synthetic textured polarimetric SAR images.
\end{abstract}

Index Terms-Polarimetric SAR images, Fisher distribution, Texture, KummerU, Classification, Segmentation.

\section{INTRODUCTION}

Synthetic Aperture Radar (SAR) data are the result of a coherent imaging system that produces the speckle noise phenomenon. The intensity and phase of the backscatter signal are modeled by a complex Gaussian distribution. For multilook one channel SAR data, the intensity follows a Gamma distribution. For multi-look multichannel Polarimetric SAR (PolSAR) data, the covariance matrix should be used. For fully developed speckle, the covariance matrix follows the complex Wishart distribution [1]. These distributions, that characterize the speckle noise phenomenon, have been generally used for SAR data analysis. It is assumed that land cover backscatter characteristics are homogeneous (uniform or not textured) over the area. This is not the case for forest areas, for example. For textured scenes, the "product model" has been proposed [2]. The observed signal is the product of a positive scalar texture component $\mu$ with the speckle component. For polarimetric SAR data, this model assumes that the texture component is independent of the polarization. The texture term is generally modeled by a Gamma distribution. The observed signal then follows a $\mathcal{K}$ distribution. This model has been largely used and seems particularly appropriate for forest areas. Others distributions (Weibull) or approximations to the $\mathcal{K}$ distribution have been proposed.

L. Bombrun is with the GIPSA-Lab, Grenoble Institute of Technology, 38031 Grenoble, France (e-mail: lionel.bombrun@gipsa-lab.inpg.fr)

J.-M Beaulieu is with the Computer Science and Software Engineering Department, Laval University, Québec City, QC, G1K-7P4, Canada (e-mail: jean-marie.beaulieu@ift.ulaval.ca)

Manuscript received November 7, 2007; revised March 29, 2008.
More recently, second-kind statistics (or Log-statistics) have been used and seem to be particularly appropriate to take into account the multiplicative aspect of speckle noise. For one channel SAR data, the Fisher distribution is used to model the observed signal for the classification of urban areas [3]. The distribution provides good approximations of different land cover types.

This paper shows how the Fisher distribution could be used to model the observed signal for multichannel polarimetric SAR data of textured scenes. The Fisher distribution is used to model only the texture component $\mu$. We first present the Wishart distribution for homogeneous areas and introduce the product model. In section III, we derive the distribution of the observed covariance matrix for Fisher distributed texture. The new pdf involves KummerU function. We examine the texture histogram of an urban area to show the advantage of this approach. Finally, we apply the new pdf in a segmentation experiment on synthetic data with varying texture distributions.

\section{COVARIANCE MATRIX DISTRIBUTION}

\section{A. Pdf for homogeneous scenes}

One-look Polarimetric SAR data are completely characterized by the scattering matrix $\mathbf{S}=\left[\begin{array}{ll}S_{h h} & S_{h v} \\ S_{v h} & S_{v v}\end{array}\right] . S_{h v}$ is the scattering component for horizontally polarized transmitting antenna and vertically polarized receiving antenna. For the monostatic case, the reciprocity assumption holds. The cross-polarization terms $S_{h v}$ and $S_{v h}$ are equal. The radar backscattering signal is described by the target scattering vector $\mathbf{x}=\left(S_{h h}, S_{v v}, \sqrt{2} S_{h v}\right)^{T}$ where $\mathrm{T}$ is the transposition operator. For homogeneous (no textured) scene (denoted by subscript $\mathrm{h}$ ) and fully developed speckle, the pdf of $\mathbf{x}$ follows a zero mean multivariate complex Gaussian distribution [1]. The L-look covariance matrix, $\mathbf{Z}_{h}=\frac{1}{L} \sum_{k=1}^{L} \mathbf{x}_{k} \mathbf{x}_{k}^{H}$, follows the complex Wishart distribution [1]

$$
p_{\mathbf{Z}_{h}}\left(\mathbf{Z}_{h} \mid \Sigma_{h}\right)=\frac{L^{L p}\left|\mathbf{Z}_{h}\right|^{L-p} \exp \left\{-L \operatorname{tr}\left(\Sigma_{h}{ }^{-1} \mathbf{Z}_{h}\right)\right\}}{\pi^{\frac{p(p-1)}{2}} \Gamma(L) \cdots \Gamma(L-p+1)\left|\Sigma_{h}\right|^{L}}
$$

where $\Sigma_{h}=E\left[\mathbf{x x}^{H}\right]$ is the population covariance matrix. $E[\cdot], \operatorname{tr}(\cdot)$ and $|\cdot|$ are respectively the expectation, the trace and the determinant operator. The superscript $H$ denotes the complex conjugate transposition. $p$ is the dimension of the target scattering vector $\mathbf{x}$ ( $p=3$ for the reciprocal case).

\section{B. The product model}

For textured areas, the observed covariance matrix $\mathbf{Z}$ can be written as the product of a texture parameter $\mu$ with 
the covariance matrix for homogeneous surface, $\mathbf{Z}=\mu \mathbf{Z}_{h}$. Generally the texture is polarimetric dependent and $\mu$ is represented by a matrix. But in this Letter, $\mu$ is assumed to be a positive scalar parameter. The probability density function of the covariance matrix $\mathbf{Z}$ can be derived by the following equation [2] [4] [5]:

$$
p_{\mathbf{Z}}\left(\mathbf{Z} \mid \Sigma_{h}, \alpha\right)=\int_{0}^{\infty} p_{\mathbf{Z}_{h}}\left(\mathbf{Z} \mid \mu \Sigma_{h}\right) p_{\mu}(\mu \mid \alpha) d \mu
$$

where $\alpha$ is the parameter set of texture distribution.

For forest areas, the gamma distribution has been widely used to model the texture. The associated covariance matrix follows a $\mathcal{K}$ distribution, so named because it uses the modified $\mathrm{K}-\mathrm{Bessel}$ function of the second kind [4].

With high resolution images of man-made objects, the Fisher distribution has been successfully introduced to model the SAR clutter [3]. In next section, the advantage of this distribution family for texture modeling is examined and the covariance matrix pdf is derived analytically.

\section{SCENE TEXTURE DISTRIBUTION}

\section{A. Fisher distribution}

The pdf of the Fisher distribution is defined by 3 parameters as:

$$
p_{\mu}(\mu)=\frac{\Gamma(\mathcal{L}+\mathcal{M})}{\Gamma(\mathcal{L}) \Gamma(\mathcal{M})} \frac{\mathcal{L}}{\mathcal{M} m} \frac{\left(\frac{\mathcal{L} \mu}{\mathcal{M} m}\right)^{\mathcal{L}-1}}{\left(1+\frac{\mathcal{L} \mu}{\mathcal{M} m}\right)^{\mathcal{L}+\mathcal{M}}}
$$

with $\mathcal{L}>0, \mathcal{M}>0$. The Fisher distribution is not related to electromagnetic wave theory. It involves the Mellin transform and second kind statistics.

\section{B. Benefit of Fisher distribution}

The ability of Fisher distribution to model real data is examined. An 8-look L-band polarimetric SAR image $(50 \times 50$ pixels) is extracted from an urban area of the Oberpfaffenhofen test site. Pixel texture values are computed by maximum likelihood estimation ( [5], see Eq. 20). For each pixel, the texture component $\mu$ is estimated from the observed pixel covariance matrix $\mathbf{Z}$ and the estimated homogeneous covariance matrix of the segment. The texture histogram is approximated by Gamma and Fisher pdf. The Fisher parameters are estimated from both moment and log-moment values (see [3] or [6] for cumulants statistics). Fig. 1 shows the real data histogram with Gamma and Fisher approximation curves.

The Fisher distribution curves give the best estimate of the texture histogram. Indeed, Fisher distributions are Pearson VI solutions and cover a large range of distributions [3] [6]. It is not confined to urban scenes. It fits reasonably forested and agricultural fields. Parameters $\mathcal{L}$ and $\mathcal{M}$ control the comportment of the head and tail of the distribution. Fisher distribution is equal to the Mellin convolution of a Gamma distribution by an inverse Gamma distribution. Fisher distribution can be viewed as a generalization of Gamma distributions. In this example, the log-moment estimation method provides a better estimate than the moment based method [3] [6].

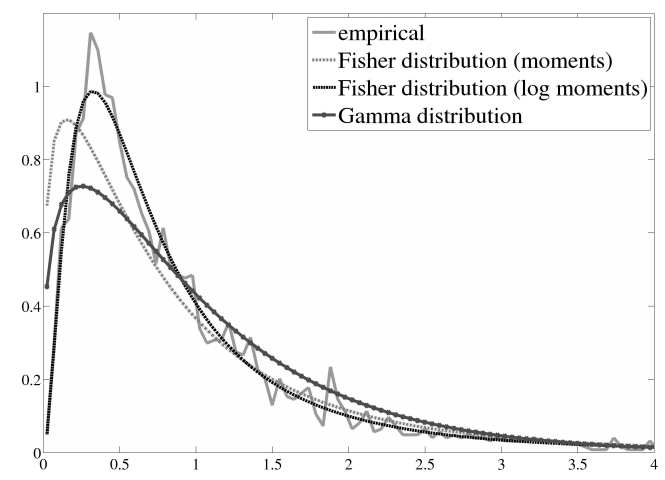

Fig. 1. Texture modeled by Gamma and Fisher pdf

\section{KummerU distributed covariance matrix}

As the Fisher distribution fit well a large range of SAR clutter [3], it is of great interest to derive the covariance matrix pdf for a Fisher distributed texture. The pdf shown in Eq. 4 (at the top of the next page) is obtained by substituting Eq. 1 and Eq. 3 inside Eq. 2.

By moving out of the integral the terms independent of $\mu$, the integral term becomes:

$$
I=\int_{0}^{\infty} \frac{\exp \left\{-\frac{L}{\mu} \operatorname{tr}\left(\Sigma_{h}{ }^{-1} \mathbf{Z}\right)\right\}\left(\frac{\mathcal{L} \mu}{\mathcal{M} m}\right)^{\mathcal{L}-1}}{\mu^{L p}\left(1+\frac{\mathcal{L} \mu}{\mathcal{M} m}\right)^{\mathcal{L}+\mathcal{M}}} d \mu
$$

If we replace the texture variable $\mu$ by $t=\frac{\mathcal{M} m}{\mathcal{L}} \frac{1}{\mu}$ in Eq. 5 ,
get: we get:

$I=\left(\frac{\mathcal{L}}{\mathcal{M} m}\right)^{L p-1} \int_{0}^{\infty} \frac{\exp \left\{-\frac{L \operatorname{tr}\left(\Sigma_{h}{ }^{-1} \mathbf{Z}\right) \mathcal{L}}{\mathcal{M} m} t\right\} t^{L p+\mathcal{M}-1}}{(1+t)^{\mathcal{L}+\mathcal{M}}} d t$

Abramowitz and Stegun have shown the following relation [7, Eq. 13.2.5] which links an integral to the confluent hypergeometric function of the second kind (KummerU, denoted by $U(\cdot)$ in Eq. 7).

$$
\Gamma(a) U(a, b, z)=\int_{0}^{\infty} \exp (-z t) t^{a-1}(1+t)^{b-a-1} d t
$$

with $\Re(a)>0$ and $\Re(z)>0$.

Next, by using the substitution $z=\frac{L \operatorname{tr}\left(\Sigma_{h}{ }^{-1} \mathbf{Z}\right) \mathcal{L}}{\mathcal{M} m}, a=$ $L p+\mathcal{M}$ and $b=1+L p-\mathcal{L}$ in Eq. 6 and by combining Eq. 4, Eq. 6 and Eq. 7; it can be demonstrated that the covariance matrix for a Fisher distributed texture has a pdf that uses a KummerU function (see Eq. 8 at the top of the next page). In the following, such pdf will be named KummerU distribution.

\section{HIERARCHICAL SEGMENTATION}

Once the covariance matrix pdf for a Fisher distributed clutter is found, criterion can be easily derived for segmentation 


$$
\begin{aligned}
& p_{\mathbf{Z}}\left(\mathbf{Z} \mid \Sigma_{h}, \mathcal{L}, \mathcal{M}, m\right)=\int_{0}^{\infty} \frac{L^{L p}|\mathbf{Z}|^{L-p} \exp \left\{-\frac{L}{\mu} \operatorname{tr}\left(\Sigma_{h}{ }^{-1} \mathbf{Z}\right)\right\}}{\pi^{\frac{p(p-1)}{2}} \Gamma(L) \cdots \Gamma(L-p+1) \mu^{L p}\left|\Sigma_{h}\right|^{L}} \frac{\Gamma(\mathcal{L}+\mathcal{M})}{\Gamma(\mathcal{L}) \Gamma(\mathcal{M})} \frac{\mathcal{L}}{\mathcal{M} m} \frac{\left(\frac{\mathcal{L} \mu}{\mathcal{M} m}\right)^{\mathcal{L}-1}}{\left(1+\frac{\mathcal{L} \mu}{\mathcal{M} m}\right)^{\mathcal{L}+\mathcal{M}}} d \mu \\
& p_{\mathbf{Z}}\left(\mathbf{Z} \mid \Sigma_{h}, \mathcal{L}, \mathcal{M}, m\right)=\frac{L^{L p}|\mathbf{Z}|^{L-p}}{\pi^{\frac{p(p-1)}{2}} \Gamma(L) \cdots \Gamma(L-p+1)\left|\Sigma_{h}\right|^{L}} \frac{\Gamma(\mathcal{L}+\mathcal{M})}{\Gamma(\mathcal{L}) \Gamma(\mathcal{M})}\left(\frac{\mathcal{L}}{\mathcal{M} m}\right)^{L p} \Gamma(L p+\mathcal{M}) U(a, b, z)
\end{aligned}
$$

or classification of PolSAR data. In this paper, the hierarchical segmentation algorithm proposed by Beaulieu and Touzi [8] is adapted to the KummerU distributed covariance matrix. The segmentation process can be divided into three steps:

1) Definition of initial partition.

2) For each 4-connex segments pair, the stepwise criterion, $\mathrm{SC}$, is computed. Then, we find and merge the two segments which minimize the criterion.

3) Stop if the maximum number of merges is reached, otherwise go to step 2 .

\section{A. Stepwise criterion}

The criterion used in the hierarchical algorithm is based on the $\log$-likelihood function. If $S_{i}$ and $S_{j}$ are two adjacent segments, the stepwise criterion $\left(\mathrm{SC}_{i, j}\right)$ is expressed as [8]:

$$
\mathrm{SC}_{i, j}=\operatorname{MLL}\left(S_{i}\right)+\operatorname{MLL}\left(S_{j}\right)-\operatorname{MLL}\left(S_{i} \cup S_{j}\right)
$$

where $\operatorname{MLL}(\cdot)$ is the segment maximum log-likelihood function.

1) For the Wishart distribution: the stepwise criterion is derived from Eq. 1:

$$
\begin{aligned}
\mathrm{SC}_{i, j}= & L\left(n_{i}+n_{j}\right) \ln \left|C_{S_{i} \cup S_{j}}\right|-L n_{i} \ln \left|C_{S_{i}}\right| \\
& -L n_{j} \ln \left|C_{S_{j}}\right|
\end{aligned}
$$

where $C_{S_{i}}$ is the mean covariance matrix calculated over the $n_{i}$ pixels of the segment $S_{i}$. It is the best likelihood estimated of $\Sigma$ for the $S_{i}$ segment.

2) For the KummerU distribution: the maximum loglikelihood function for segment $S$ is derived from Eq. 8. After removing terms that will be cancelled in the stepwise criterion, the log-likelihood function can be rewritten as (see Eq. 11 at the top of the next page).

$\hat{\mathcal{L}}, \hat{\mathcal{M}}$ and $\hat{m}$ are respectively the estimated of the Fisher parameters $\mathcal{L}, \mathcal{M}$ and $m$ by the log-cumulants method [3] [6]. $C_{h}$ is the best likelihood estimate of $\Sigma_{h}$ for the segment $S$.

\section{B. Segmentation of a synthetic image}

The hierarchical segmentation algorithm proposed by Beaulieu and Touzi [8] has been implemented with the KummerU criterion (Eq. 11). For real data, the between region variation of the covariance matrix is often more important than the variation of the texture distribution. We use a synthetic image to consider only texture distribution variations. Fig. 2(b) shows the simulated texture image $(200 \times 200$ pixels $)$. It contains four $100 \times 100$ Fisher realizations generated by the

\begin{tabular}{|c|c|}
\hline texture 1 & texture 2 \\
$\mathcal{L}=5$ & $\mathcal{L}=5$ \\
$\mathcal{M}=10$ & $\mathcal{M}=30$ \\
$m=1$ & $m=1$ \\
& \\
\hline texture 3 & texture 4 \\
$\mathcal{L}=10$ & $\mathcal{L}=10$ \\
$\mathcal{M}=10$ & $\mathcal{M}=30$ \\
$m=1$ & $m=1$ \\
& \\
\hline
\end{tabular}

(a)

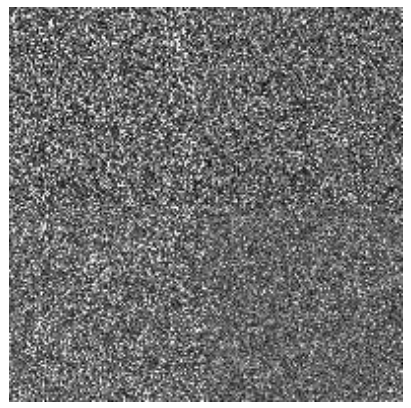

(b)

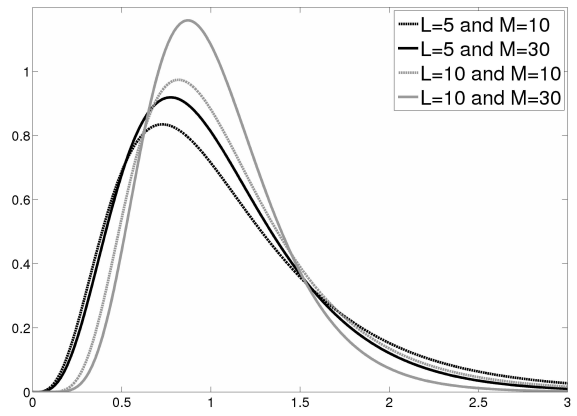

(c)

Fig. 2. (a) image containing the 4 segments (ground truth) and Fisher parameters used in the simulation (b) 4 -areas synthetic texture image $(200 \times 200)$, (c) Fisher distributions used for the simulation

inversion of the cumulative repartition function. Those Fisher distributions are presented in Fig. 2(c). The speckle is constructed from a Wishart distribution with the same parameters over the whole image (8-look). Wishart samples are generated using the algorithm proposed by Odell and Feiveson [9]. Then, the PolSAR dataset is generated by multiplying the texture image by the homogeneous covariance matrix (speckle). The segmentation algorithm is executed with an initial partition where each segment is a bloc of $10 \times 10$ pixels. The initial partition is composed of 400 segments.

Tab. I shows the Kolmogorov distance between the Fisher distributions used in the simulation. Note that this distance is rather small (from 0.049 to 0.102 ). Segmentation of the simulated data is a difficult task because the same Wishart distribution is used and texture are quite similar, as can be observed in Fig. 2(b) 


$$
\begin{aligned}
\operatorname{MLL}(S)= & -n L \ln \left|C_{h}\right|+n \ln \{\Gamma(\hat{\mathcal{L}}+\hat{\mathcal{M}})\}-n \ln \{\Gamma(\hat{\mathcal{L}})\}-n \ln \{\Gamma(\hat{\mathcal{M}})\}+n L p \ln \left(\frac{\hat{\mathcal{L}}}{\hat{\mathcal{M}} \hat{m}}\right) \\
& +n \ln \{\Gamma(L p+\hat{\mathcal{M}})\}+\sum_{\mathbf{z}_{k} \in S} \ln \left\{\mathrm{U}\left(L p+\hat{\mathcal{M}} ; 1+L p-\hat{\mathcal{L}} ; \frac{L \operatorname{tr}\left(C_{h}{ }^{-1} \mathbf{Z}_{k}\right) \hat{\mathcal{L}}}{\hat{\mathcal{M}} \hat{m}}\right)\right\}
\end{aligned}
$$

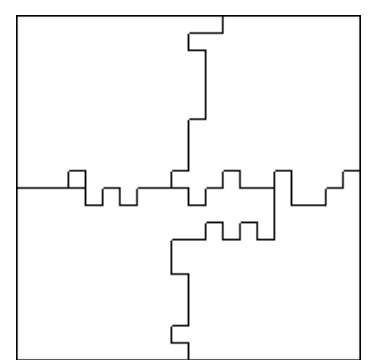

(a)

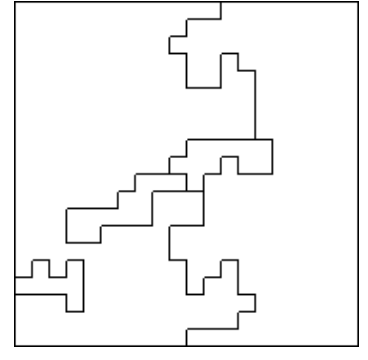

(b)

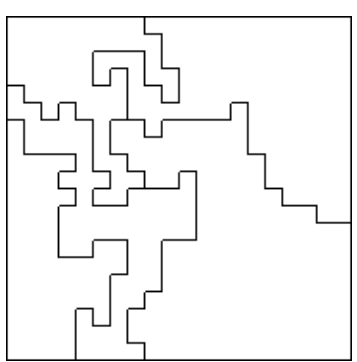

(c)

Fig. 3. Partitions with 5 segments produced by (a) KummerU criterion, (b) Wishart criterion, (c) $\mathcal{K}$ criterion

TABLE I

KOLMOGOROV DISTANCE BETWEEN THE 4 TEXTURES.

\begin{tabular}{|c|c|c|c|c|c|c|}
\hline \hline textures & $1-2$ & $1-3$ & $1-4$ & $2-3$ & $2-4$ & $3-4$ \\
\hline$d_{K}$ & 0.049 & 0.074 & 0.102 & 0.063 & 0.092 & 0.072 \\
\hline \hline
\end{tabular}

\section{Segmentation result}

The segmentation result with 5 segments is shown in Fig.3. For comparison, the result obtained from the Wishart and the $\mathcal{K}$ distribution based criteria are presented [8]. The best result is obtained with the KummerU criterion.

The evaluation of segmentation result is a difficult problem. Fig.4 presents curves similar to Receiver Operator Characteristic (ROC) curves that clearly show the advantage of the KummerU criterion. For a pixel $x$, let $S_{x}$ be the set of pixels belonging to the same output segment as $x$ and let $T_{x}$ be the set of pixels belonging to the same input ground truth region as $x . C_{x}$ is the complement of $T_{x}$ and $|T|$ is the size of the set $T$ (the number of pixels in the set). For each pixel $x$, we define the detection ratio as $\frac{\left|S_{x} \cap T_{x}\right|}{\left|T_{x}\right|}$ and the false alarm ratio as $\frac{\left|S_{x} \cap C_{x}\right|}{\left|C_{x}\right|}$. By taking the average values of these ratios over the whole image, we obtain measures corresponding to the detection probability $p_{d}$ and the false alarm probability $p_{f a}$. The segmentation process starts with a

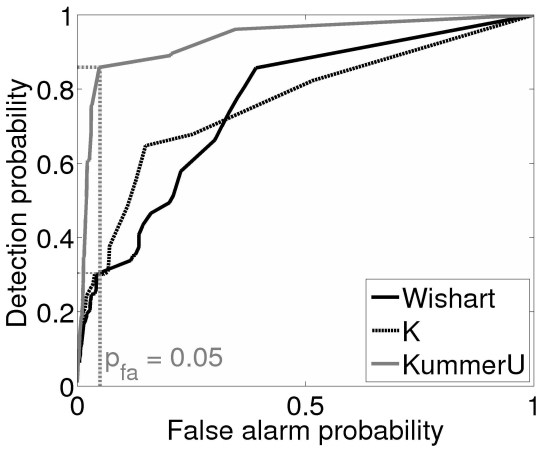

Fig. 4. ROC curves

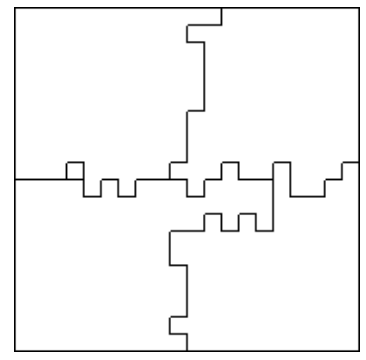

(a)

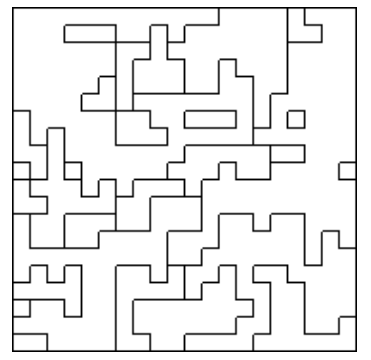

(b)

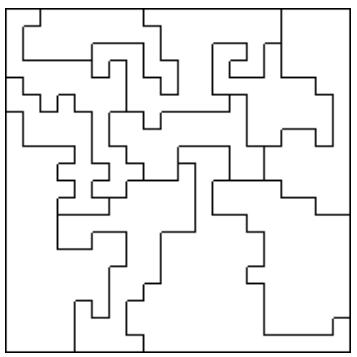

(c)

Fig. 5. Segmentation for a given false alarm probability $p_{f a}=0.05$ for (a) KummerU criterion (5 segments), (b) Wishart criterion (29 segments), (c) $\mathcal{K}$ criterion (11 segments)

partition of 400 segments. At each iteration, a new partition is produced by merging 2 segments. The ROC curves are obtained by calculating $p_{d}$ and $p_{f a}$ for each partition.

In Fig. 4, the ROC curve for the KummerU distribution is better than the curves for the Wishart distribution and $\mathcal{K}$ distribution. When $p_{f a}=0.05$, the detection probability is much better for the KummerU criterion (0.85) than for the Wishart and $\mathcal{K}$ distribution criterion (0.3) and the corresponding partitions, shown in Fig.5, contain respectively 5, 29 and 11 segments. This shows the advantage of using a criterion that can discriminate between a large range of texture distributions. 


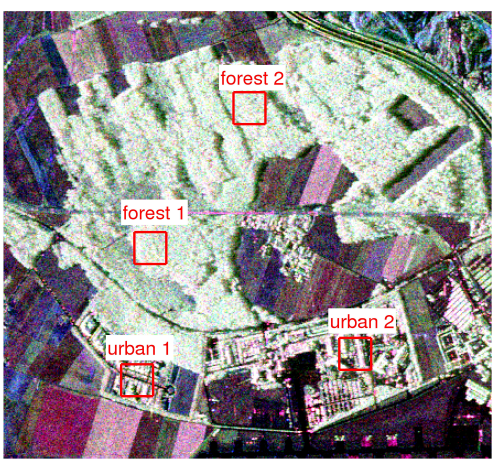

Fig. 6. 2 forest areas and 2 urban areas (30x30 pixels) are selected into the Oberpfaffenhofen image.

\section{Criterion comparison with real data}

In real application, the speckle covariance matrix difference is often more important than the texture difference. This would seriously limit the advantage of the KummerU distribution. The evaluation of the benefit of the KummerU distribution in real application is an involved task. We present a simple case with 2 forest areas and 2 urban areas (30x30 pixels) manually selected into the Oberpfaffenhofen image (Fig. 6). The presented criteria are calculated for each region pair (Table. II). A small value indicates that the 2 regions of the pair are similar. For the Wishart distribution, the distance between the 2 urban regions (10290) is larger than the difference between any urban region and any forest region. This indicates that the Wishart criterion will have difficulty to separate urban areas from forest areas. This is not the case for the $\mathcal{K}$ and KummerU distribution where the urban1-urban2 distance (340 for $\mathcal{K}$ and 68 for KummerU) is smaller than the urbanforest distances. The KummerU criterion considers the 2 urban regions more similar than do the $\mathcal{K}$ criterion (68 vs 340 ). This will facilitate the grouping of urban regions.

Forest 1 and Forest 2 regions are equally similar, independent of the texture model employed $(\mathrm{SC} \simeq 200)$. Texture appears unimportant for these forest classification.

Moreover, the KummerU distribution can tends to merge segments from different class. The Forest1-Forest2 criterion is only twice lower than the Urban1-Forest1 criterion for the KummerU distribution, whereas this ratio is about 3 for the $\mathcal{K}$ distribution.

\section{CONCLUSiON}

In this paper, the efficiency of using a Fisher distribution to model the texture of polarimetric SAR data has been shown. Then, with the product model hypothesis, the covariance matrix distribution for a Fisher textured clutter has been derived. The pdf has been mathematically established and expressed as a function of the confluent hypergeometric function of the second kind (KummerU). Next, this distribution has been implemented on a hierarchical segmentation algorithm by calculating the log-likelihood function. Comparisons with the Wishart and $\mathcal{K}$ distributions have been done for synthetic textured scenes. ROC curves reveal that the use of an appropriate
TABLE II

BETWEEN REGION SIMILARITY COMPARISON FOR THE WISHART, THE $\mathcal{K}$ DISTRIBUTION AND THE KUMMERU DISTRIBUTION

\begin{tabular}{|r|r|r|r|}
\hline \hline & Urban 1 & Urban 2 & Forest 1 \\
\hline Wishart distribution & & & \\
\hline Urban 2 & 10290 & & \\
\hline Forest 1 & 6850 & 7178 & \\
\hline Forest 2 & 6826 & 7238 & 201 \\
\hline $\mathcal{K}$ distribution & & & \\
\hline Urban 2 & 340 & & \\
\hline Forest 1 & 645 & 1221 & \\
\hline Forest 2 & 609 & 975 & 199 \\
\hline KummerU distribution & & & \\
\hline Urban 2 & 68 & & \\
\hline Forest 1 & 471 & 1499 & \\
\hline Forest 2 & 436 & 1244 & 200 \\
\hline \hline
\end{tabular}

texture distribution is useful to segment textured polarimetric SAR images.

A complete analysis of the interplay between polarimetric variability and scalar texture must be carried out to have a better understanding of the segmentation algorithm performances.

Future developments could involve the combination of Wishart and KummerU based criterion for the segmentation of mixed images with textured and no textured areas. An other perspective of this work could be the adaptation of the Wishart classifier to the KummerU distribution [10].

\section{ACKNOWLEDGMENT}

The authors would like to thank the anonymous reviewers for the constructive comments and corrections that helped to improve this letter.

\section{REFERENCES}

[1] N. Goodman, "Statistical analysis based on a certain multivariate complex Gaussian distribution (an introduction)," in Ann. Math. Statist., vol. 34, 1963, pp. 152-177.

[2] I. Joughin, D. Winebrenner, and D. Percival, "Polarimetric Density Functions for Multilook Polarimetric Signatures," IEEE Transactions on Geoscience and Remote Sensing, vol. 32, no. 3, pp. 562-574, 1994.

[3] C. Tison, J.-M. Nicolas, F. Tupin, and H. Maître, "A New Statistical Model for Markovian Classification of Urban Areas in High-Resolution SAR Images," IEEE Transactions on Geoscience and Remote Sensing, vol. 42, no. 10, pp. 2046-2057, October 2004.

[4] J. Lee, D. Schuler, R. Lang, and K. Ranson, "K-Distribution for MultiLook Processed Polarimetric SAR Imagery," in Geoscience and Remote Sensing, IGARSS '94, Pasadena, California, United States, 1994, pp. 2179-2181.

[5] A. Lopès and F. Séry, "Optimal Speckle Reduction for the Product Model in Multilook Polarimetric SAR Imagery and the Wishart Distribution," IEEE Transactions on Geoscience and Remote Sensing, vol. 35, no. 3, pp. 632-647, 1997.

[6] J.-M. Nicolas, "Application de la transformée de Mellin: étude des lois statistiques de l'imagerie cohérente," in Rapport de recherche, 2006D010, 2006.

[7] M. Abramowitz and I. Stegun, Handbook of Mathematical Functions With Formulas, Graphs, and Mathematical Tables, 1964.

[8] J.-M. Beaulieu and R. Touzi, "Segmentation of Textured Polarimetric SAR Scenes by Likelihood Approximation," IEEE Transactions on Geoscience and Remote Sensing, vol. 42, no. 10, pp. 2063-2072, 0ctober 2004.

[9] P. Odell and A. Feiveson, "A numerical procedure to generate a sample covariance matrix," Journal of the American Statistical Association, vol. 61, no. 313, pp. 199-203, 1966.

[10] J. Lee, M. Grunes, T. Ainsworth, L. Du, D. Schuler, and S. Cloude, "Unsupervised Classification Using Polarimetric Decomposition and the Complex Wishart Classifier," IEEE Transactions on Geoscience and Remote Sensing, vol. 37, no. 5, pp. 2249-2258, 1999. 